\title{
Evidence Based Patient Education to Promote Natural Childbirth Experiences: Educating your Patients to know the Right Questions to ask to meet their Own Personal Birthing Needs
}

\author{
Erica Sisiam* \\ Lecturer of Nursing, Lander University, School of Nursing, Greenwood, SC, USA
}

"Corresponding author: Erica Sisiam, Lecturer of Nursing, Lander University, School of Nursing, Greenwood, SC 29649, USA; Tel: 864.388.8278; Fax: 864.388.8122; E-mail: esisiam@lander.edu

Received date: Apr 06, 2015; Accepted date: Apr 23, 2015; Published date: May 1, 2015

Copyright: (C) 2015 Sisiam E. This is an open-access article distributed under the terms of the Creative Commons Attribution License, which permits unrestricted use, distribution, and reproduction in any medium, provided the original author and source are credited.

\section{Abstract}

Nurses need to educate and advocate for women seeking a natural un-medicated childbirth experience. Evidence based practices are difficult to research for a nurse and are often overly complicated preventing application to the average individual's personalized birthing plan. Increasing educational support and available alternatives allows for informed decision making, and promotes a natural childbirth, which directly improves patient satisfaction. Education needs to start from the point of antenatal care, prior to entering a birthing facility. Hospitals and birthing centres that have more options and alternatives to support natural un-medicated childbirth can additionally increase revenue by becoming a destination facility.
\end{abstract}

Keywords: Evidenced based practices; Un-medicated childbirth; Education; Informed decisions; Patient satisfaction; Birthing plans

\section{Background}

Women have options available to them to create their preferred labour experience, and can select either a natural un-medicated labour experience or an experience involving medication(s). Informed consent and knowledge of available options is necessary to guide individualized "birthing plans" and personal preferences to meet personal birthing needs. In recent years hospitals have seen an increased number of patients seeking an un-medicated or natural birth experience as opposed to using epidural anaesthesia [1]. Women who initially desired a natural childbirth experience, but were unable to follow through with their desire by requesting an epidural, rate their overall experience as less satisfying. This is despite getting pain relief as opposed to the individuals who followed through with a natural childbirth experience [2]

\section{Discussion}

Even healthcare professionals who are confident in their own ability to retrieve research information, interpret methodologies in journals, and feel comfortable searching for online resources can feel challenged when attempting to utilize online evidence based resources. The sheer magnitude of information and the time consuming action of searching does not give a simple answer that healthcare professionals can directly apply to a simple clinical scenario [3]. In labor, delivery, and post-partum inpatient units, the primary front line staff caring for these families are nurses, with ancillary staff including doulas and midwives. Organizational and individual factors are associated with the incorporation of evidence based practice within a facility, as administration needs to provide a culture of encouragement to staff to develop policies that promote use of current literature [4]. With healthcare professionals being challenged in finding, and incorporating evidence based literature into practice, how much more daunting is it for the lay individual seeking out medical services and care to find the best treatment or even alternative choices to meet their own individual need and preferences? How can we drive our patients to the destination of the birth experience that they want? Nurses need to be proactive in educating and ensuring informed decision making of the birthing experience. Often women find "birthing plans" online and fill in the blanks with their desires, but do they fully understand these options, or even know the capabilities of the facility? Other pregnant individuals do not enter into the laboring facility with a specific plan but expect to be walked through the entire process, trusting those who care for them.

With consumer centered healthcare some patients are very engaged in making their own healthcare decisions, but large vulnerable populations exist including patients who are in pain, require fast paced care, or who are in a busy nursing department [5]. Actions of healthcare professionals, invasive technology, and standardized procedures and physician orders, all decrease the perceived involvement and sense of control in the labor process, therefore decreasing maternal satisfaction with the experience [6]. Additionally, the use of teachable coping strategies can directly affect the intensity of pain felt by an individual [7]. A labouring woman on an inpatient labor ward can have multiple criteria which place her at risk of a decreased satisfaction and increased pain. Early education can best serve the pregnant woman as she can identify her preferences early, and when she is in increasing labor pain affecting her ability to learn, her ability to seek out information and absorb it, will be decreased. Education ideally, should start while the woman is at her routine antenatal doctor appointments. Flyers, pamphlets, and packets given by the office or obstetric counsellor can contain information associated with the individual physicians practice and how this relates to the local hospital(s) capabilities, and allow the individual to contemplate their preferential options and needs. With Lamaze and childbirth education classes being an out of pocket expense, many women seek a self-education methodology using research on the internet as an available option. Hospital and childbirth center 
education programs can be provided based on a fee or scholarship to meet the needs of the population. Facility capabilities should be clearly posted or found in an admission packet made available upon request or from an obstetrical office.

\section{Linking Evidence to Action}

Healthcare professionals need to encourage proper informed decisions by presenting all of the available options including the benefits and risks of those options, to allow for the best possible personalized birth experience. Nurses and other healthcare professionals are initiators of change and can promote the normalcy of the labor process and labor options to decrease unnecessary interventions which can increase the likelihood of medical complications [9]. Western culture has "medicalized" the birth experience into a structured hospital procedure, but innovative facilities are accommodating a woman's right to choose preferential options that are safe evidence-based alternatives [10]. Hospitals offering increased preferential options may increase the revenue for the hospital by becoming a favored destination in the area [10].

Knowing the available options allows the individual to plan a desired direction for the course of their labor, make family/loved ones aware of facility policies and personal preferences, and be mentally prepared knowing that many alternatives exist if one item is not working when the labor experience occurs. Knowledge surrounding the labor process alleviates fear of the unknown. Nurses and other healthcare professionals need to educate women on preferential options or ensure women know to ask about some of the following evidence based or consumer based questions.

- What are your visitation hours?

- What is your limit of individuals in the room throughout labor and during delivery?

- Do you have any policies regarding the role of a doula or midwife not employed by the facility?

- What are your nurses to patient ratios?

- What is your policy on eating, drinking, and intravenous (IV) access?

- What is your policy on ambulating with rupture of membranes (broken water)?

- Do you provide telemetry for remote fetal monitoring allowing for ambulation during labor or do you have a policy on the frequency of auscultation of fetal heart tones?

- Do you have birthing balls, rocking chairs, massage equipment, and birthing stools?

- Do you offer herbal products as an alternative choice or to aid in the labor process?

- Are you equipped to perform water-births?

- Will your staff allow me to and work with me using Lamaze breathing, vocalizations, or chanting?

- Do you offer breastfeeding support or classes?

- Do have a policy on or provide therapeutic showers?

- Do you have a policy on or provide sterile water injections?

- When do you typically clamp the umbilical cord (after the physiological stage three)?
- Do you allow post-delivery kangaroo mother care?

\section{Conclusion}

Evidence-based practice supports continuous care from a nurse, midwife, or lay support person provides positive maternal and newborn results including decreased use of medications, decreased length of labor, decreased need for equipment use, medical interventions, decreased caesarean section rates, increased newborn assessment scores, and increased maternal satisfaction [11]. Healthcare professionals need to advocate for evidence-based policy change within a hospital system to revise practices to include more complimentary modalities to support an un-medicated natural labor [10] and promote community awareness on available options or programs. Allowing a more customer-made labor experience will increase the autonomy of the individual, support women seeking an un-medicated labor, decrease complications/interventions, and increase satisfaction of the labor experience. Knowing the policies, limitations, and available options could only increase satisfaction scores for a facility, increasing referrals in the community to the facility and increasing the attractiveness of the facility. Education, informed decisions, and increased available evidenced based options create an optimal satisfying birth experience, better patient outcomes, and facility revenue.

\section{References}

1. Condra, T. M., Hafendorfer L (2011) Inspiring and Leading Women to Succeed at Unmedicated Childbirth. JOGNN: Journal Of Obstetric, Gynecologic \& Neonatal Nursing, 40: S5-6.

2. Kannan S, Jamison RN, Datta S (2001) Maternal satisfaction and pain control in women electing natural childbirth. Reg Anesth Pain Med 26: 468-472.

3. Isetta, M. (2008). Evidence-based practice, healthcare delivery and information management: A contemporary case study, Aslib Proceedings 60: $619-641$.

4. Boström, A., Rudman, A., Ehrenberg, A., Gustavsson, J., and Wallin, L. (2013). Factors associated with evidence-based practice among registered nurses in Sweden: a national cross-sectional study. BMC Health Services Research 13:165.

5. Johnstone MJ (2011) Nursing ethics and informed consent. Aust Nurs J 19: 29.

6. Benoit C, Zadoroznyj M, Hallgrimsdottir H, Treloar A, Taylor K (2010) Medical dominance and neoliberalisation in maternal care provision: the evidence from Canada and Australia. Soc Sci Med 71: 475-481.

7. Smith CA, Levett KM, Collins CT, Jones L (2012) Massage, reflexology and other manual methods for pain management in labour. Cochrane Database Syst Rev 2: CD009290.

8. Smith CA, Levett KM, Collins CT, Crowther CA (2011) Relaxation techniques for pain management in labour. Cochrane Database Syst Rev : CD009514.

9. Romano AM, Lothian JA (2008) Promoting, protecting, and supporting normal birth: a look at the evidence. J Obstet Gynecol Neonatal Nurs 37: 94-104.

10. Harper B, (2009) Helping women achieve what they want in a hospital birth: Education not alienation. International Journal of Childbirth Education 24:10-13.

11. Medves J (2002) Review: continuous caregiver support during labour has beneficial maternal and infant outcomes. Evid Based Nurs 5: 105. 